Voix et Images

voixetimages

\title{
Lecture structurale d'Alain Grandbois : le poème 18 des îles de la nuit
}

\section{Yves Laliberté}

Volume 1, numéro 1, septembre 1975

Hubert Aquin

URI : https://id.erudit.org/iderudit/013988ar

DOI : https://doi.org/10.7202/013988ar

Aller au sommaire du numéro

Éditeur(s)

Les Presses de l'Université du Québec

ISSN

0318-9201 (imprimé)

1705-933X (numérique)

Découvrir la revue

\section{Citer cet article}

Laliberté, Y. (1975). Lecture structurale d'Alain Grandbois : le poème 18 des Îles de la nuit. Voix et Images, 1(1), 89-105. https://doi.org/10.7202/013988ar d'utilisation que vous pouvez consulter en ligne.

https://apropos.erudit.org/fr/usagers/politique-dutilisation/ 


\section{Lecture structurale d'Alain Grandbois : le poème 18 des îles de la nuit}

Le mode d'analyse adopté par le critique dépend en grande partie de la conception qu'il se fait de la poésie. Il y a peut-être autant de méthodes de "démonter" une pièce qu'il y a de définitions du principe poétique. Croit-on que l'art des Saint-Denys Garneau et des Grandbois s'explique par leur philosophie, leur «vision du monde», on recourt à la recherche thématique, et c'est logique! Au contraire, l' "essence " du poème est-elle sécrétée par le signifiant seul? On aura alors droit à une étude formaliste. Le structuraliste, loin d'être associé à cette dernière tendance, garde plutôt un juste milieu puisqu'il ne dédaigne ni le sens ni la forme. Comment voit-il la poésie, et comment la distingue-t-il de la prose, par exemple? Laissons à André Martinet le soin de donner une première réponse: "la forme poétique [...] transfigure le mot et en fait tout autre chose que ce qu'il est dans la prose de la vie quotidienne " ${ }^{\prime}$. Voilà un des aspects par lequel l'énoncé poétique réclame une place à part au sein de la littérarité: la spécificité du rapport signifiant/signifié. Grandbois, imposant sa vision du monde, les signifiants se distendent pour loger ses connotations personnelles. Le signe "morte", par exemple, acquiert une valeur favorable, par-delà les idiosyncrasies d'une civilisation matérialiste, au moment où, pour le poète, la mort devient le seuil d'une délivrance. Cette loi de la poésie à laquelle le poéticien doit se conformer, MM. Delas et Filliolet l'expriment en ces mots dans leur ouvrage récent Linguistique et poétique: "la sélection s'effectue en fonction de l'actualisation du sens propre au texte et non par la prise en compte du référent ${ }^{2}$ ".

Mais c'est sans doute Jean Cohen qui légitime le mieux la méthode structurale lorsqu'il introduit un nouveau type de rapport: "la poésie est un langage motivé » et «un langage motivé, c'est toujours un rapport de ressemblance entre les trois couches relativement autonomes de la langue, la syntaxe, le sens et le $\operatorname{son}^{3}$ ". Cohen suppose donc que, par-delà le simple rééquilibrage des deux articulations, la poésie entend établir une corrélation très marquée entre les structures syntaxiques, sonores, sémantiques et - on pourrait ajouter - visuelles. Le parallélisme de ces diffé- 
rents niveaux avait été souligné à l'origine par Roman Jakobson dans la célèbre définition de la structure poétique qu'il entendait comme la «projection du principe d'équivalence de l'axe de la sélection sur l'axe de la combinaison ${ }^{4}$. De ce parallélisme, ou équivalence, doit naître une macrostructure, la communication hyper-organisée du poème, sa raison d'être. (D'ailleurs, le roman moderne qu'on qualifie de roman-poème n'est-il pas caractérisé justement par un souci de la structuration voulue signifiante par elle-même?) Le code particulier ainsi engendré par le poème va être déchiffré par la lecture structurale qui a, comme dirait Benveniste, la "conscience du système ${ }^{5}$ ".

II s'agit donc pour nous de dégager les analogies que le poème 18 des îles de la nuit suggère entre la formalisation du message (ce que Chomsky appelle la "performance") et les desseins de ce message (la "Compétence") puisque "la tâche essentielle du poéticien est de montrer comment l'encodeur s'est servi d'une matière, la langue, pour élaborer un modèle de performance, une réalisation particulière ${ }^{6} \%$.

Notre étude ne se propose pas d'épuiser toutes les potentialités du poème non plus qu'elle ne se targue d'une objectivité positiviste. À l'instar des théoriciens, nous acceptons le risque qui est, en fait, un postulat heuristique, d'être sollicité par un code plutôt que par un autre. Dans ce cas, la rigueur pallie avantageusement une «archilecture" qui, de toute façon, demeure au rang des chimères. Notre sélection d'éléments dits "pertinents" devra être conditionnée par une possibilité d'intégration à l'intérieur d'une des nombreuses structures en puissance dans le poème. Un choix qui semblera parfois arbitraire ne perdra donc jamais de vue la découverte ultime, et rassurante, du fonctionnement global.

Afin de minimiser les préjugés résultant d'une expérience pré-textuelle de Grandbois, nous éviterons de situer le poème dans une veine thématique. Le poème 18 , objet de notre analyse presque par hasard, sera amené à se dire dans son individualité en admettant à priori l'isolement de ses quatorze vers. Au départ, cette sorte de maïeutique ignore le taux de "réceptivité structuraliste» du corpus (à cet égard, il faudra admettre la moindre pertinence de la face sonore), emprunté tel qu'il apparaît dans le recueil des Poèmes d'Alain Grandbois publié aux Éditions de l'Hexagone ${ }^{7}$.

II

A 01 Le rêve s'empare de son doux visage de morte

02 Un miraculeux brouillard l'élève et la transporte

03 Au delà des régions dévorées par le temps

B $04 \quad$ Cet invisible et tendre feu plus vivant que le sang

05 Elle le nourrit de sa paix la plus profonde 


\begin{tabular}{|c|c|c|}
\hline \multirow{5}{*}{ C } & $\begin{array}{l}06 \\
07\end{array}$ & $\begin{array}{l}\text { Ses doigts ont écarté l'épouvante du monde } \\
\text { Et baignée de songes ainsi que l'Archange } \\
\text { sacré } \\
\text { Elle sourit enfin d'un sourire délivré }\end{array}$ \\
\hline & 09 & $\begin{array}{l}\text { si le grand rivage absorbe ses petites } \\
\text { mains charnelles }\end{array}$ \\
\hline & 10 & $\begin{array}{l}\text { si le flux de la mer balaie ses larmes } \\
\text { mortelles }\end{array}$ \\
\hline & 11 & $\begin{array}{c}\text { Ah si l'éclair aveugle jusqu'au sable } \\
\text { de la nuit }\end{array}$ \\
\hline & 12 & $\begin{array}{l}\text { Ses blessures nous échappent comme un } \\
\text { cristal attendri }\end{array}$ \\
\hline
\end{tabular}

D $\quad 13$ Car elle est le cœur et la vie et la porte

14 Du secret retrouvé dans son refuge de morte

III

Voyons d'abord comment le poème se prête à une première démarcation des séquences. Le travail pour trouver les "grandes divisions" ne se fait pas au jugé quoiqu'il démarre sur une base apparemment très faillible, l'impression immédiate que les sens ont du poème. La lecture structurale se fraie un chemin à travers l'objet d'étude depuis l'écorce jusqu'au cœur, pourrait-on dire en langage imagé, interrogeant d'abord ce que nous appellerons la structure superficielle, c'est-à-dire l'appareil graphique et la trame sonore, pour passer ensuite à la structure profonde, syntaxe et sémantique.

Les formes visuelles, perçueś simultanément, précèdent l'interprétation des segments, discursifs par définition. Le poéticien suit donc un itinéraire naturel quand il répond d'abord aux stimuli provoqués par les marques externes et en fait ses repères explicatifs à priori. En ce qui nous concerne, il faut envisager avec réserve la présentation graphique, sinon l'on risque de s'engager sur un terrain mouvant. A défaut de la pagination originale, force nous est de passer outre certains traits «d'architecture " qu'une analyse structurale ne saurait éluder. Le caractère typographique et le rejet, par exemple, sont des phénomènes qui portent à confusion puisqu'ils ne sont pas les mêmes d'une édition à une autre. En revanche, nous prendrons pour acquis que les strophes et les quatorze vers (c'est-à-dire les retours à la ligne marqués par la majuscule), évidemment plus résistants aux remaniements, caractérisent la spatialisation primitive du poème, celle de Grandbois.

Trois surfaces à la dimension verticale équivalente $(1,5 \mathrm{~cm}$ chacune dans l'édition de l'Hexagone) organisent l'espace pertinent des pages 55 et 56: ce sont les blancs qui délimitent quatre blocs syntagmatiques. Les strophes ainsi générées proposent quant à elles une forme initiale composée de quatre séquences. Ces masses visuelles correspondent-elles vraiment à la matrice structurale du poème? Si oui, cela ne va pas de soi. En poésie moderne, l'équilibre formel n'est pas appréhendé de façon 
contraignante comme en poésie traditionnelle. Si le sonnet, par exemple, implique nécessairement un parallélisme inhérent aux strophes initiales et terminales, impaires et paires, extérieures et intérieures, la disposition des vers libres de Grandbois ne présente pas ces équivalences automatiques "quant au genre ${ }^{8}$ ". Des indices linguistiques plus que des règles conventionnelles de versification suggèrent le ou les patterns latents.

Au premier coup d'œil, le poème présente une physionomie sévère, sans grand artifice syntaxique. Cette impression nous vient d'abord du découpage des strophes en blocs syntaxiques autonomes. On ne nous permet pas ce bond' vif de la voix et de la pensée d'une strophe à l'autre comme au poème $21^{9}$, par exemple, où l'expansion des syntagmes circonstanciels participe au moulage des formes:

Devant ces bûchers fraternels

Malgré les cendres inutiles

Et malgré cette fuite d'ailes

Malgré la morte odeur des villes

S'est dressé le seul Arbre d'Or...

Ici, une proposition relativement simplifiée se termine sans enjambement. Parce qu'elle est complète à l'intérieur de la strophe, la syntaxe tend à conférer à cette strophe une valeur iconique: correspondraient aux quatre zones visuelles des totalités sémantiques apparemment différentes.

La strophe finale du poème 18 revendique à cet égard un statut particulier, non qu'elle fasse exception à l'autonomie strophique, quoi qu'on en pense. La conjonction "car" n'établit pas un rapport de dépendance entre un «terme inférieur» et un «terme supérieur» (Tesnière ${ }^{10}$ ). L'indépendante des vers 13 et 14 se contente d'ajouter une notion causale, antérieure au verbe «échapper» du vers 12; où elle se fait remarquer, c'est dans la chute arithmétique de sa figure spatiale ( $A$ : trois vers; $B$ : cinq vers; $C$ : quatre vers; $D$ : deux vers). Pourtant le blanc entre les vers 12 et 13 propose une équivalence entre le segment des vers $13+14$ et les segments des vers $01+02+03(D-A)^{11}, 04+05+06+07+08(D-B)$ et $09+10+11+12(D-C)$ respectivement. II faut donc supposer que l'entité visuelle $D$, concomitante à l'achèvement spatial, est, en quelque sorte, inversement proportionnelle aux unités sémantiques en $D$, hypersignifiantes afin que soit rééquilibré le rapport strophique. Et, en effet, - comme nous le verrons plus tard - un contenu particulièrement dense caractérise l'achèvement sémantique. L'influence de $D$ est d'autant plus grande que, coupée de la principale, elle s'offre en corrélat de $A$ et $B$ tandis que $C$ apparaît elle-même comme corrélat de $D$.

II convient d'interroger, à ce stade-ci de notre étude, l'axe vertical des paradigmes. Avec lui, nous plongeons au niveau de la structure profonde du poème, la «mise en perspective ${ }^{12}$ " sémantique, seule capable de ratifier les structures partielles saisies au gré de la spatialisation et de la syntaxe. Ce faisant, il n'est pas certain qu'un premier schème de séquences, fortement empirique, disons-le, soit retenu comme étant le 
plus fonctionnel (quoique, à la limite, toute organisation reste pertinente, en puissance, par-delà le choix de codifications du poéticien).

L'hypothèse des quatre strophes-séquences, posée comme premier jalon herméneutique, reçoit jusqu'à un certain point l'appui du lexique dans ce qu'il a de plus grammatical. En effet, on constate que les actants (Tesnière ${ }^{13}$ ), qui regroupent les deux tiers des substantifs et pronoms, sont distribués de façon à nouer une relation originale à chaque strophe. Ces actants s'inscrivent tous sans exception à l'intérieur de paradigmes plus ou moins étendus selon le cas. Les catégories sont coiffées des étiquettes archilexiques suivantes: le «Elle», le «Rêve», l' «Abstrait», la «Nature» et l' «Ego humain» (c'est-à-dire le «nous» de l'actant-narrateur associé à un veuvage universel), ou plus particulièrement sans doute l' «Ego viril». Voici l'aspect que rend l'actualisation actantielle, de sorte que toutes les strophes s'opposent par un de leurs protagonistes:

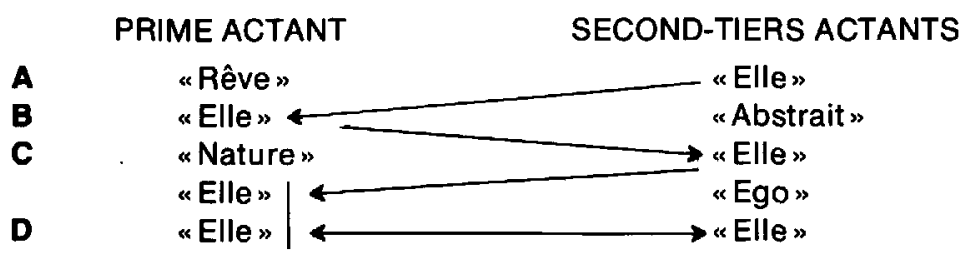

Vu que les actants de «Rêve" et de “Nature» sont exclusifs, ils légitiment ce jeu de logicien: $A\lrcorner C \perp B, A\lrcorner C\lrcorner D$, laissant pour seule équation possible $B-D$. Cependant, à cause du verbe copule «être» en D, le paradigme de l' "Abstrait ", auquel appartiennent par emploi métaphorique les mots «cœur», «vie» et «porte», commute avec celui du «Elle». On vérifie donc, par le biais, le particularisme de la dernière strophe, elle qui accorde à l'être féminin une "surprésence". Le poème se termine dans la résolution du polémos actant vs actant puisque l'attribut se fondant au sujet, tout est écho de ce dernier, et non plus patient distinct (05) ou antagonisant (06).

II est clair que la récurrence des formes référentielles du "Elle" le place au sommet hiérarchique des paradigmes. Son emploi de dénominateur commun donne d'ailleurs lieu à des analogies susceptibles de briser l'isolement artificiel des strophes. Le tableau ci-dessus a le mérite de capter la façon dont le poème organise l'ambivalence du «elle", utilisé tour à tour comme agent et comme patient (chassé-croisé indiqué par les flèches). Des couplages sont patents entre $A$ et $09+10+11$ de $C$, entre $B$ et $12+13+14$. Or, le rythme positionnel de l'actant-thème (pour adapter à la situation le concept "mot-thème" de Pierre Guiraud) donne le signal d'un revirement de la trame narrative selon que la femme agit (prime actant) ou, à l'opposé, selon qu'elle connaît une période de passivité réceptive (second et tiers actants). Les couplages évoqués plus haut se doublent donc, au plan sémantique, du rôle commun que les paires de segments concernées font jouer à la femme dans «l'histoire" du poè- 
me. Même si l'équivalence semble se borner à cette symétrie sémantique, gardons en tête les deux cycles binaires qu'elle imprime à la pièce: 1. Subit + Agit; 2. Subit + Agit. Peut-être se révéleront-ils les infrastructures de séquences plus vastes que les strophes.

Nous avons mentionné à juste titre l'importance du «elle» dans le poème. II apparaît comme un invariant sémantique auquel se rattache un réseau référentiel hiérarchisé par divers rapports. La représentation schématique suivante identifie ces rapports:

ELLE

\begin{tabular}{|c|c|c|c|c|}
\hline Pronominal & Corrélatif & Comparatif & Métaphorique & Synecdochique \\
\hline & & & & (non figuré) \\
\hline$l^{\prime}-\mathrm{la}(02)$ & $\begin{array}{l}\text { blessures } \\
\text { (12) }\end{array}$ & $\begin{array}{l}\text { Archange } \\
(07)\end{array}$ & cœur (13) & visage (01) \\
\hline elle (05) & nuit (11) & & vie (13) & doigts (06) \\
\hline elle (08) & & & - porte (13) & sourire (08) \\
\hline elle (13) & & & & mains (09) \\
\hline & & & & larmes (10) \\
\hline
\end{tabular}

Les référents les plus usités, pronominaux et synecdochiques, sont en conséquence les indices privilégiés du sens. Dès le premier vers, par exemple, la notation d'un physique, «doux visage de morte», sous-entend l'intervention féminine dans le poème. Pour qui a lu les lles de la nuit, la figure synecdochique ne surprend guère puisque la femme se manifeste constamment sous la forme de parties du corps. Mais, au poème 18, la sélection des termes, "visage»(01), «doigts»(06), «mains"(09), “larmes"(10), "sourire" (08) - et non bouche - vise à connoter une relation purement platonique sans aucune référence à la copulation, voire à la passion. La rupture phatique appuie cette absence d'intimité physique. L'emploi de la troisième personne grammaticale «elle» au lieu de la seconde "tu" ou du "nous" inclusif est la marque d'une distanciation entre le locuteur et l'être évoqué. Quelle est la raison de cette réserve? Les pronoms personnels répondent en quelque sorte à cette question à chaque fois qu'ils réitèrent dans le cours de l'énoncé, en tant qu'anaphoriques, la définition contextuelle de la femme. On sait que "l'information sémantique dont sont porteurs ces pronoms est égale à la somme des informations données préalablement dans le texte par les segments qu'ils reprennent ${ }^{14} \%$. Or, le seul sème prédicatif constituant cette somme d'informations n'est autre que "morte"(01); livré à l'amorce du poème, cet état apparaît donc comme principe initial rendant le développement ultérieur possible et nécessaire. Ainsi, les pronoms "elle" et «la" expliquent la séparation évoquée par d'autres procédés linguistiques quand ils véhiculent le sème “morte» en leitmotiv, du moins jusqu'à la dernière strophe 
où va s'opérer une "surqualification" (Cohen ${ }^{15}$ ) concomitante à une réorientation du message.

Le vide sémique créé par l'absence du générique "femme" et par le rejet d'une connaissance pré-textuelle de l'actant principal suffirait à mettre l'emphase sur le sème de la mort. Mais, en plus, sa distribution symétrique aux extrémités du poème l'institue dans des fonctions de commande sémantique. La focalisation qui, dès le premier vers, se fait sur "morte" en tant que diagnostic de l'état intrinsèque - si l'on peut dire de la femme reste vivace puisque le constat revient en conclusion. Du fait que "morte" encercle le poème, tout semble d'abord en partir puis y converger comme si le poème se refermait telle une boucle.

$\mathrm{Ce}$ noyau de signification génère diverses séries paradigmatiques dont la tâche est de donner une image des conséquences de la mort. A cet égard, le dictionnaire Quillet-Grolier fournit un prédicat qui s'avère le foyer d'un premier ensemble sémique: “Dans le langage ordinaire, [l'espace] se dit d'une étendue limitée et superficielle." Le vers 03 fait appel à ces notions de limites et d'espace défini avec la connotation du lexème "régions". La classe de substitution ainsi formée par ce seul élément, quoique l'on puisse intégrer le sème “temps" à un paradigme extensif (espace limité + temporalité), s'oppose dès lors aux unités à venir, "grand rivage»(09), "mer»(10), "éclair»(11), et surtout, " refuge de morte" (14), qui, d'une part, connotent un espace métaphysique sans frontières perceptibles et, d'autre part, transmettent à degré zéro une information temporelle. La dichotomie (espace limité + temporalité) vs (espace illimité + atemporalité) s'actualise donc aux deux niveaux, du signifié d'abord, et du rapport inverse qui lie à la mort les unités des deux paradigmes en opposition. Car, en effet, la lecture globalisante du poème re-situe le contraste dans une structure supérieure qui prend la forme suivante: MORT」 (espace limité + temporalité), vu que la locution prépositive dans:

Au delà des régions dévorées par le temps

installe une négation entre ce paradigme et la femme morte, tandis que MORT - (espace illimité + atemporalité), pose la détermination épithétique dans "refuge de morte" rendant compte d'une identité corrélative entre la mort et un espace-asile intellectuel impliqué par "refuge".

Imprécise et non mesurable, la notation qui caractérise tout le poème sert aux mêmes fins que le paradigme du "Rêve "; comme les éléments synonymiques, "rêve" (01), "songes" (07) et métonymique, «brouillard " (02), elle a pour rôle d'infuser une atmosphère irréelle au poème en même temps que s'opère la déréalisation de l'actant “elle» sous l'action des agents oniriques. Par l'allégorie du "Rêve", Grandbois imagine la mort de la femme en une vision d'assomption qui a tout d'une transformation heureuse et souhaitable. La seule vertu dénotative des épithètes relatives au paradigme du "Rêve" fait entrer les sèmes qui le composent dans l'isotopie surjacente des "Positifs" ou, encore, des "Euphoriques". Par exemple, les adjectifs dans "doux visage"(01) et «sourire délivré"(08) traduisent le soulagement et la sérénité consécutifs à un phénomène li- 
bérateur. Dans cette optique, le «miraculeux» du vers 02 est particulièrement intéressant vu son antéposition au substantif: cet écart par rapport à l'emploi général ${ }^{16}$ ne semble pas motivé dans ce cas par un souci d'euphonie mais plutôt par le désir de conférer du relief à une connotation figurée de l'adjectif, celle de merveilleux et d'étonnant (QuilletGrolier), qui vient faire l'unanimité autour d'un sentiment de félicité.

En parlant tantôt "d'isotopie surjacente", nous soupçonnions d'ores et déjà que le caractère mélioratif de certains sèmes ne se voulait pas l'apanage d'une classe de connotation. II semble en effet que les contenus émotifs engendrent une distinction apte à englober toute la verticalité du poème, de sorte que le sémantisme se fractionnerait en substance "euphorique», correspondant aux adjuvants de la mort, d'une part, et, d'autre part, en substance "dysphorique», accolée au monde de contingences. Les concepts entre parenthèses que nous faisons nôtres ont été introduits à la critique par A. J. Greimas dans son étude Du sens où il formule I'hypothèse théorique suivante:

S'il est incontestable que la communication poétique est, dans son ensemble, créatrice d'euphorie, il ne fait pas de doute que la substance phonétique et sémantique qui est l'objet de cette communication se trouve connotée, au niveau de toutes ses articulations, par la catégorie proprioceptive manifestant tantôt son terme euphorique, tantôt son terme dysphorique ${ }^{17}$.

En ce qui nous concerne, le trait le plus significatif de cette taxinomie réside dans la prédominance des termes dits «euphoriques". Livronsnous quelques instants à un relevé statistique. Une démarche «quantifiante ", peu opérable au niveau de paradigmes restreints à quelques éléments par le corpus choisi, éclaire par contre la distribution d'un «affect " présent dans chaque substantif, verbe ou adjectif, quelle que soit sa classe sémantique. Sur cinquante-deux signes marqués, trente-huit suggèrent une valeur positive, compte tenu des connotations personnelles de Grandbois ; ce qui abandonne à la série des «dysphoriques" vingt-sept pour cent seulement des occurrences pertinentes [«régions"(03), «dévorées»(03),

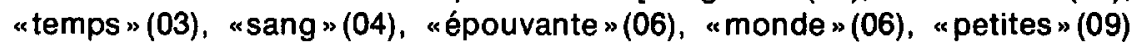
— opposé à «grand»-, «mains»(09), «charnelles»(09), "larmes»(10), “ mortelles »(10), «sable»(11), «nuit»(11), «blessures»(12)]. Toutefois, le rapport mathématique $38 / 14$ demeure en deçà de la vérité du poème puisqu'il ne reflète pas l'effectivité véritable des sèmes en contexte narratif. Ainsi, une collusion des mécanismes linguistiques réduit de façon définitive l'impact négatif déjà affaibli par une carence signalétique: un seul terme se trouve en tête de vers ( «blessures"), un seul joue le rôle de prime actant ( «blessures"), un autre seulement est agent, c'est-à-dire "qui cause" lato sensu ( "temps"); le soixante-treize pour cent d'éléments positifs accapare donc les postes privilégiés laissés vacants. Enfin, sur le plan de la signification, les signes négatifs subissent les neutralisations consécutives de la locution "au delà"(02), de la conjonction «plus... que»(04) et des verbes privatifs «ont écarté »(06), «absorbe»(09), «balaie»(10), «aveugle»(11) et «échappent»(12). Finalement, des qua- 
torze signes négatifs, il n'en reste aucun affirmant une permanence, une présence de force.

Dans le seul but d'illustrer les règles restrictives du structuralisme théorique, disons qu'une autre interprétation du texte, “impressionniste" celle-là, verrait à greffer une continuité phonétique à ce phénomène sémantique. Il serait alors permis de suivre le raisonnement syllogistique suivant: $1^{\circ}$ La face acoustique du poème accorde la précellence aux consonnes latérales [1] et [r], tant au chapitre des occurrences totales (37 et 41 respectivement, contre 25 pour leur plus proche concurrent, le [s]), qu'au chapitre de la fréquence relative dans chaque strophe; $2^{\circ}$ or, un symbolisme phonétique voit dans la musicalité douce et agréable de ces consonnes l'expression d'une certaine sérénité ; $3^{\circ}$ cette nappe sonore reflète donc un climat de quiétude dont la précellence a déjà été enregistrée au niveau du sens. Cette conclusion intéressante, et parfaitement justifiée dans l'analyse dite “traditionnelle ${ }^{18}$ " reste irrecevable pour les théoriciens Delas et Filliolet qui, par ces mots, reprennent à leur compte la position de Roman Jakobson:

Ce qui compte, ce n'est pas tant l'impression donnée par le sens, impression d'ailleurs flottante et considérablement modifiée par le contexte (le $[r]$ intervocalique de [iris] est évidemment fort éloiané du [r] final de [ã:Kr], que la possibilité offerte par cette substance, lorsqu'elle est utilisée de façon autonome, de créer des échelles de correspondances hiérarchisées ${ }^{19}$.

Notre habileté à utiliser le signifiant sonore s'en trouve paralysée de beaucoup. Pour rester fidèle à la méthode, nous ne pouvons prétendre que les latérales ont un effet lénitif sur le poème, les phonèmes ne signifiant pas par eux-mêmes. En revanche, on peut s'attacher aux similarités possibles entre leur distribution et la distribution du sens. Par exemple, le retour des rimes riches en [o:rt] aux deux premiers et deux derniers vers consacrera un rapport d'équivalence entre ces segments, ce rapport ayant d'abord été pressenti à travers le parallélisme positionnel et la référence réciproque à la Cause motrice du poème, la mort, mentionnée à ces seuls endroits. Mais revenons à nos paradigmes «euphoriques» et “dysphoriques $* . .$.

L'extinction du sème “dysphorique» qu'on remarquait tantôt se trouve être la résultante de la situation narrative, le poème 18 se situant à la frontière entre deux états antagonistes dont l'un est en régression. En effet, l'unicité du poème semble se faire autour d'une structure supérieure qui engloberait le clivage «euphoriques»/ «dysphoriques». Se résumant ainsi : “État nouveau (qui s'impose) vs “État ancien» (qui agonise), cette matrice structurale est à la base d'un réseau d'oppositions d'abord diffuses au sein des deux premières strophes. Malgré la distance syntagmatique les séparant, un Au-delà présumé au vers 03 entre en conflit avec le «monde» du vers 06; la «paix» du vers 05 contraste nettement avec "l'épouvante" du vers suivant. Quand le temps dévore, la femme nourrit - et, ici, la venue de "l'État nouveau" est traduite sur une tout autre partition, un sème de prodigalité succédant à un sème de spoliation. Au 
vers 04 , la comparaison «feu plus vivant que le sang "supplée à l'absence des procès évolutifs et privatifs et, à leur place, entretient une opposition, cette fois-ci entre "sang " et «feu». L'antinomie en est arrivée à un pointclé de sa mission descriptive puisqu'elle dévoile la psyché de ces états en guerre, leurs principes vitaux à tous deux. D'une part, le sang est indice de vie, c'est-à-dire qu'un rapport vécu lie son signifié à l'existence terrestre telle que nous la connaissons. Rendue possible, la substitution de termes équivalents "sang " - "vie " dans l'équation "feu " vs "sang " débouche, d'autre part, sur "feu" $\downarrow$ "vie", puis, finalement, sur "mort" vs "vie", du fait que, à cause de l'épithète "tendre", le "feu» rallie le paradigme des "euphoriques", lui-même associé à "l'État nouveau " de la mort. Le segment du vers 04 préside donc à un resserrement notable du message poétique puisque son contenu connoté nomme la vie et la désigne, elle et ses attributs, comme étant cet autre pôle opposé à la mort. II est maintenant clair que Grandbois met en lice le sort terrestre et le destin eschatologique. Ce dernier, le domaine de "l'euphorique ", instaure en plus une nouvelle qualité d'être qui ne se veut pas soumise à la matérialité altérable du sang. Le feu suppose que la morte "vit sa mort»ou, si l'on préfère, "vit sa vie de l'Au-delà " grâce à l'énergie dispensée par un flux artériel igné. Voulu "invisible", ce principe vital du feu s'apparente aux images déréalisantes du "Rêve " et de "l'Espace illimité "; comme elles, il consacre la sublimation de la femme.

La tension signifiante du vers 04 pouvait déjà être prévue à travers son arrangement syntaxique. Trois facteurs suffisent à mettre le segment en évidence et à marquer une instance sémantique: le déplacement, le démonstratif et l'absence de verbe.

1. LE DÉPLACEMENT. Commun dans la langue parlée, le déplacement du complément d'objet direct est rare dans la langue écrite et comporte toujours son effet. On en dénombre huit de la sorte dans les îles de la nuit qui viennent conférer une importance accrue au groupe nominal

Cet invisible et tendre feu plus vivant que le sang

Voici l'objet privilégié et, avec lui, un souci de caractérisation trahi par les épithètes coordonnées. Dans le cas du comparatif "plus vivant que le sang", le déplacement vient pallier en quelque sorte une relativisation possible, mais absente dans tout le poème. D'ailleurs, pour des fins de prosodie peut-être, Grandbois n'abuse guère de la relative dans ses poèmes: sa présence plus que son absence surprend le lecteur et... signifie. La commutation qui préfère "plus vivant que le sang" à "qui est plus vivant que le sang" fait que substantif et adjectif «restent, comme disent MM. Delas et Filliolet, à l'intérieur d'unités syntagmatiques en contiguïté ${ }^{20}$ " . Cela sert le mot "feu» dont le prestige n'est pas amoindri par la subordination.

2. LE DÉMONSTRATIF. Le démonstratif trône en tête de vers et, par le fait même, au début de la strophe. Le "cet" (et avec lui les "ce", "cette" et "ces") a une trop longue histoire dans les îles de la nuit pour 
dédaigner la moindre de ses apparitions. Souvent, par le passé, Grandbois a multiplié le démonstratif afin de soutenir par l'emphase du ton les aspirations prométhéennes du thème ${ }^{21}$. Ici, le présentatif isolé donne, certes, plus d'ampleur au discours mais il pointe d'abord le doigt sur le substantif qui, à nouveau, se retrouve sur la sellette.

3. L'ABSENCE DE VERBE. Dans un poème où foisonne le verbe, l'isolement d'un syntagme non verbal double la valeur sémantique déjà suggérée par le déplacement. Les pauses, limitrophes du vers, forcent le lecteur à embrasser toute l'étendue conceptuelle du symbole avant que de le voir associé à une action et anémié par les radiations verbales. Le "feu" prend donc pour Grandbois une hypersignifiance, imité en cela de l'apposition du vers 07 (on décèle quand même l'idée d'un procès derrière l'adjectif verbal), du circonstanciel du vers 03 et du déterminant du vers 14. La pertinence de ces deux dernières occurrences souffre toutefois de l'orthodoxie de la postposition.

Loin d'être développé au niveau d'un syntagme restreint, le pattern bâti à partir du duel entre les sèmes de vie et les sèmes de mort reste pertinent au-delà des deux premières strophes, d'autant plus que la dichotomie atteint une acuité jamais réalisée sauf au vers 06 . Les vers 09,10 et 11 , s'ils perpétuent la transition vie/mort, la systématisent en quelque sorte par l'alignement de constructions jumelles (sujet + verbe + complément d'objet direct); la cohésion syntaxique appelle une triple égalité sémantique, du moins dans l'ordre d'un rapport annihilant qu'entretiennent sujets et patients. Chaque vers se présente donc comme un diptyque aux volets antithétiques:

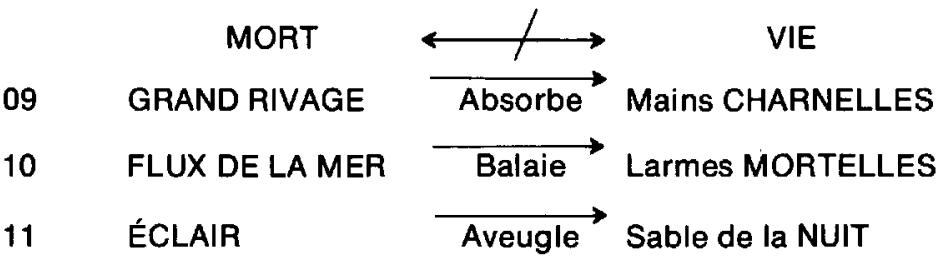

Cette opposition vie/mort aura été une mine sémique puisqu'elle féconde encore d'autres images. Cette fois-ci, Grandbois sollicite le registre naturel et ce sont les agents d'une certaine géographie qui, à travers le symbolisme poétique, jouent les rôles d'exterminateurs d'un monde imparfait. Jetons un coup d'œil à cette série de contrastes en C: «grand rivage» vs "petites mains charnelles", «mer" (infinie) vs «larmes" (gouttes), "éclair" (phénomène titanesque qui dépasse la dimension humaine) vs "sable de la nuit ". La disproportion entre antagonistes, nettement accusée par les signes et les connotations que nous avons soulignés à dessein, entend grossir le paradigme de la mort des sèmes de «démesure" et de "puissance" tandis que l'empire de la vie, suggéré par les adjectifs "charnelles» et “mortelles», paraît victime d'une force irrésistible. Une autre constatation non moins intéressante est la non-équivalence qui s'immisce 
au sein des référents synecdochiques de la femme. Indissociables à prime abord (voir tableau p. 11), on voit se former deux factions bien distinctes selon que le physique appartient à une femme déjà morte ou, au contraire, se rapporte à un être encore rivé à l'attraction du réel. Ainsi, "visage de morte»(01) - «doigts"(06) - «sourire»(07) ل « mains charnelles" "larmes mortelles", les reliquats de l'existence d'ici-bas. Cette dualité qui pose deux identités féminines, non simultanées, disons-le, provient bien sûr de la matrice binaire; mais il convient d'ajouter qu'en troisième strophe, la commutation sémantique coïncide avec l'alternance de la femme-actant qui, après avoir été patient en $A$, agent en $B$, redevient patient en $C . .$. et qui dit patient dans le poème 18 suppose un objet instable en voie de transformation. II est donc probable qu'à l'heure où s'achèvent les procès “absorbe" et “balaie", les mains et les larmes sont sensées se départir de la gangue péjorative des épithètes afin d'accéder au paradigme «euphorique» que peuplent déjà les trois autres parties du corps. Fait à noter: l'Au-delà de Grandbois n'engage pas une croyance mystique à l'abandon, par l'âme, du corps matériel ou mieux, des formes humaines. Celles-ci semblent profiter les premières de ce qui se révèle être une promotion téléologique de la femme. Toujours présentes après la mort, elles se trouvent désormais affranchies des tares terrestres.

A la lumière de ce que nous avons vu jusque-là, est-ce que le poème 18 constitue vraiment un bloc monolithique sur le plan de la signification? II semblerait en effet que la dialectique «vie vs mort» condense toute la matière, mais il faudrait pour cela compter sans le vers 12 et, surtout, sans la dernière strophe où disparaît toute trace d'antithèse. II faut plutôt revenir en arrière, réexaminer le passage de la deuxième à la troisième strophe tout en se méfiant de l'apparente continuité de sens des vers 09,10 et 11 . Laissons de côté ce simple fait et il saute aux yeux qu'à divers points de vue la troisième strophe exécute une rupture avec ce qui précède. La différence syntaxique est patente puisque la séquence $(A+B)$ s'entête à conserver l'indépendante alors que $C$ se construit plutôt par enchâssement :
A
$\begin{cases}01 & \text { Indépendante } \\ 02 & \text { Indépendante }+ \text { Indépendante } \\ 03 & \end{cases}$
$\left.\begin{array}{r}S N+S V \\ S N+(S V \\ (S V\end{array}\right\}$
$B \quad\left\{\begin{array}{cc}04 & \\ 05 & \text { Indépendante } \\ 06 & \text { Indépendante } \\ 07 & \\ 08 & \text { Indépendante }\end{array}\right.$
SV
$\mathrm{SN}+\mathrm{SV}$
SN
$(\mathrm{SN})+\mathrm{SV}$
C $\quad \begin{cases}09 & \text { Subordonnée } \\ 10 & \text { Subordonnée } \\ 11 & \text { Subordonnée } \\ 12 & \text { Principale }\end{cases}$
$(\mathrm{SN})+\mathrm{SV}$
$\mathrm{SN}+\mathrm{SV}$
$\mathrm{SN}+\mathrm{SV}$
$\mathrm{SN}+\mathrm{SV}$
$\mathrm{SN}+\mathrm{SV}$ 

D $\left\{\begin{array}{l}13 \\ 14\end{array}\right.$ Indépendante
SN + SV
SV

La nouvelle connexion va modifier radicalement, dans les vers 09,10 et 11 , le statut du pattern vie/mort qui n'est plus contemplé pour lui-même mais mis à la disposition d'un "terme supérieur" qui fait ressortir son propre contenu grâce à l'opposition entre principale et subordonnées. La lutte "vie vs mort " change donc, fonctionnellement. Dans un autre ordre d'idées, la troisième strophe contrarie l'élan binaire des deux premières et ralentit le rythme conséquemment à l'intervention du narrateur par l'interjection. Le "Ah" ne faisant pas partie de l'unité syntaxique de la subordonnée, il ne s'intègre donc pas aux anabases terminées par le

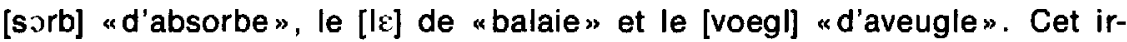
réductible réclame sa propre intonation d'autant plus que le blanc qui l'isole oblige de pauser assez longtemps pour engendrer un tiers accent tonique qui s'additionne à la dernière syllabe sonore des protases et apodoses normales. D'abord scandé par deux mouvements symétriques, le rythme du vers passe à trois temps. L'exclamation n'a pas que des répercussions prosodiques, loin de là. Elle pose d'emblée une éruption sentimentale qui fait que le poème jusque-là d'un « irréalisme objectif ", pourraiton dire, glisse vers une subjectivité de plus en plus accrue à mesure que l'anaphore annonce l'implication personnelle du poète par le "nous" au vers 12. On ne saurait assez insister sur la venue de ce pronom qui, soit dit en passant, constitue le seul élément du paradigme que nous avons appelé «l'Ego humain ». II semble être l'axe sémantique sur lequel pivotent les strophes $C$ et $D$, les distinguant par le fait même de $A$ et $B$ où il brille par son absence. L'orientation nouvelle qu'il donne au message à partir de la troisième strophe le met en droit d'introduire une seconde structure, synthétique celle-là, qui se superposerait tout en étant concomitante au patron analytique vie/mort. Là nous touchons à l'articulation globale du poème la plus satisfaisante, à notre avis, puisqu'elle ne délaisse aucun segment; des séquences $(A+B),(C+D)$, répondent le plus adéquatement aux pré-requis d'un système, pré-requis que Delas et Filliolet indiquent quand ils disent que:

Plus la fonction est forte, plus la structuration est globalisante, plus elle est intégrative, c'est-à-dire plus l'interdépendance des niveaux de fonctionnement est sentie comme première; est alors laissé au hasard (à l'arbitraire linguistique) un minimum tendant vers zéro, tout tendant à être récupéré pour signifier un certain rapport au monde ${ }^{22}$.

Cette organisation d'ensemble viendrait confirmer la forme-signe perçue au tout début de notre étude, c'est-à-dire qu'elle entérine la forme initiale des strophes mais pour l'intégrer à un second système, inclusif celui-là, qui aboutit à la forme finale $(A+B),(C+D)$. Cette structuration la plus intégrative possible découpe la substance sémantique à deux niveaux distincts; apparaissent en effet comme des marques différenciatives entre séquençes, d'une part, la dissémination sémique, entendant par là l'exclusivité de certaines images et, par-dessus tout, l'apparition de 
signifiés inédits tels, en $(C+D)$, ceux connotés par «blessures»(12) et «secret»(14), d'autre part, le registre narratif par lequel l'on a choisi de livrer le message poétique au destinataire. Primordialement parce qu'il y a le "nous" du vers 12 , la venue de la troisième strophe sur la seconde déclenche, à nos yeux, une volte-face dans l'attitude du poète face à sa matière. Grandbois est d'abord occupé à faire le RECIT de “l'assomption " postmortem de la femme puis il abandonne la dernière séquence au DISCOURS. Dans la mesure où l'on peut se représenter la chose, c'est comme si le poème prenait tout à coup les apparences d'un monologue après s'être borné à une description «du dehors".

Cela explique l'impression que donnent les deux premières strophes de se suffire à elles-mêmes. La relation du parcours d'après-mort, amorcée par une première strophe-transit, connaît une fin satisfaisante au vers 08 où l'adverbe de temps "enfin" semble le point ultime d'une chronologie discursive et consacre la finalité d'une démarche. $A$ cet égard, le passage d'un passé indéfini, "ont écarté", au présent "sourit" des vers 06 à 08 , récapitule le continuum temporel de l'évolution féminine vers la béatitude et le clôt. L'information propre au récit se résout donc de façon logique dans les limites de la séquence $(A+B)$; si cette information communiquée par le truchement d'un modèle antithétique est récupérée momentanément aux vers 09,10 et 11, cette redondance, ou "flashback" si l'on imagine une ligne temporelle au poème, sert de prétexte pour relancer l'idée directrice dans une autre direction. Comme on vient de le voir, cette autre direction se manifeste d'abord au niveau du point de vue, la conscience engagée de Grandbois prenant assez abruptement la relève d'une narration impersonnelle. Cela arrive au moment où la promotion ségoïste" de la femme doit être transposée dans une perspective universelle pour justement accuser l'exclusivité du phénomène transcendant (d'où l'absence du «nous» dans les deux premières strophes) et pour exprimer, en conséquence, la solitude de l'homme qui, lui, ne quitte pas le «monde de l'épouvante». La structure syntaxique «subordonnées vs principale" met donc en opposition directe deux effets de la mort: la chance du «elle» (subordonnées) et le malheur des autres auxquels on soustrait sa présence (principale). Voilà en définitive l'argument de la troisième strophe: pendant que la métamorphose de la femme entre dans ses diverses phases, concurremment la morte se dérobe peu à peu à son compagnon de vie, plus particulièrement au poète qui cherche à atténuer sa déception personnelle par le pluralisme du «nous". C'est le lexème «blessures" qui suggère le mal infligé, par la mort de la femme, à ceux qui restent. Ce signe que nous avons placé antérieurement dans un rapport corrélatif avec l'actant “elle», par le fait même, représente ce dernier de façon accidentelle, comme "nuit»(11), c'est-à-dire qu'il ne restitue pas le référent par l'intrinsèque ( «mains", «larmes» le font, par exemple) mais plutôt par le biais du rayonnement de la femme sur les autres. Quoi qu'il en soit, l'homologie des formes possessives exige l'équivalence, "ses mains-ses larmes-ses blessures". Par contrecoup, «blessures" adhère au champ sémantique défini à la fois par les sèmes "femme», "vie terres- 
tre» et, finalement, "femme de la vie terrestre". Il aura été dit que «la femme de la mort" n'aura laissé derrière elle qu'un souvenir douloureux de cette "femme de la vie terrestre". L'entrée en scène d'un nouveau protagoniste entraîne donc un premier contact avec l'adversité, inconnue jusque-là dans un poème gagné à l'euphorie.

Avant de passer au rôle de la dernière strophe dans la séquence $(C+D)$, il faut pour le bien comprendre soulever une autre caractéristique de la troisième strophe. En effet, il est important de noter que l'équivalence sémantique posée entre les sèmes «mains", "larmes» et "blessures" pris isolément s'accommode d'une non-équivalence sur le plan des actualisations respectives de ces signes. Le chiasme n'est pas sans modifier les contextes syntaxiques mais c'est surtout l'influence du sens verbal sur chacun des signes qui les divise. Les compléments d'objet des subordonnées disparaissent instantanément; à cet effet, l'effulguration dénotée par "l'éclair" du vers 11 montre bien la rapidité avec laquelle les seconds actants sont tous jugulés par les verbes "absorbe", "balaie" et "aveugle». Au contraire, les «blesșures" paraissent se dissiper très lentement. Le circonstant, «comme un cristal attendri», semble comparer le souvenir à un minéral qui, réduit à l'état liquide par fusion, fuit goutte à goutte, laborieusement. Pourquoi la mémoire du poète se refuse-t-elle à oublier? Et pourquoi donc la femme est-elle si importante à ses yeux que sa mort l'affecte à ce point? Tout en répondant à ces questions, les deux derniers vers en aparté prolongent le sens de la troisième strophe et substituent plus ou moins au noyau sémantique de la séquence $(A+B)$, qui avait été la mort, un nouveau foyer d'unification, une nouvelle Cause amenée par la conjonction “car». Grandbois oppose à l'identité de «morte"(01) de la femme qui lui vaut, à elle, sa propre délivrance, son autre identité, magnanime, qui, est celle, ici-bas, de trait d'union entre l'homme et sa délivrance c'est-à-dire entre l'homme et l'Absolu. Dans cette mise en lumière du rôle de la femme:

... elle est le cœur et la vie et la porte

le poème atteint les sommets de sa signification thématique puisque le poète esquisse la dialectique qui préside à la plus grande partie de son œuvre. II le fait en une sortie grandiloquente. La coordination des prédicats est syntaxe propre à infuser de l'élocutoire. Le choix de la conjonction "et", qu'on peut appeler «polysyndète» en langage technique, au lieu de la virgule ou de l'espace blanc (asyndète) vise à mettre en relief chacun des déterminants qui s'en trouvent également valorisés comme le fait voir l'arbre syntaxique suivant:

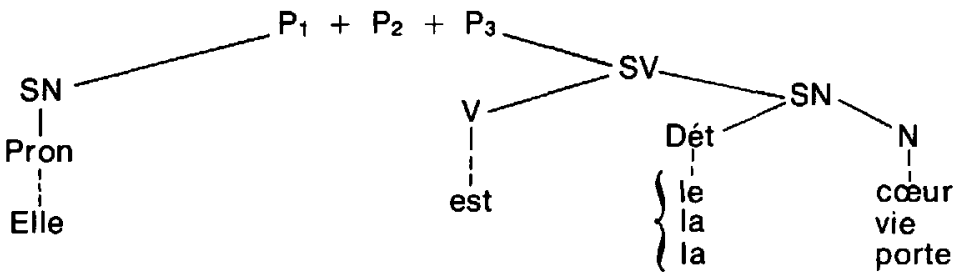


Les deux premiers termes de l'énumération incantatoire semblent se répondre dans un mouvement inductif. Cœur/vie, de la métonymie à sa résolution, l'on réitère fermement le vitalisme de la femme. Quant à l'autre terme, «porte", une de ses connotations fait appel au rôle de guide: femme-sésame qui permet de pénétrer le mystère d'un Graal surhumain. D'ailleurs, Grandbois avait déjà supposé cette vocation d'intermédiaire en comparant la femme à un "Archange sacrée au vers 07. L'image religieuse venait renforcer la solennité et le caractère sacré de l'aventure vers l'Au-delà mais n'entendait pas remplacer le matérialisme cosmologique qui prévaut sur le registre «biblique ${ }^{23}$ ", quoique ce dernier soit perceptible en fin de poème. Le préfixe itératif dans "retrouvé"(14) nous ramène en effet à la croyance d'un Ailleurs jadis possédé par l'homme. S'agit-il du paradis perdu dont le mythe a été célébré entre autres par Milton avant Grandbois? On ne peut en être sûr au poème 18 où la mort ne se manifeste que sur une dimension cosmique; quant au référent auquel renvoie le mot «secret", il reste vague et prête à la recherche un cachet d'ésotérisme. La mort n'est pas encore ce véhicule mystique vers un Dieu qui se mêlera à la thématique au poème 27 seulement ${ }^{24}$.

Mais laissons au critique thématique le soin de préciser au long des Îles de la nuit ce lieu idéal qu'anticipe le poète et les variations qui le caractérisent. Quant à nous, notre mission est dûment remplie: nous avons essayé de dégager un fonctionnement global et, à force d'indices visuels, syntaxiques, prosodiques et sémantiques, nous nous sommes aperçus qu'il était orchestré par les structures, synthétique $(A+B) \perp(C+D)$, et analytique vie vs mort qui chevauche la précédente. Ces organisations se conjuguent pour légitimer au niveau du poème 18 la définition que Jean Cohen donne de la poésie: “... la poésie est l'art de susciter par des procédés formels le contenu pathétique latent des termes de la langue et de former des énoncés motivés par l'accord de ces contenus ${ }^{25} \mathrm{~m}$.

Yves Laliberté

1. André Martinet, “Connotations, poésie et culture , dans To Honour Roman Jakobson, Paris, La Haye, Mouton, 1971, vol. II, p. 1292.

2. Daniel Delas et Jacques Filliolet, Linguistique et poétique, Paris, Larousse Université, 1973 , p. 109.

3. Jean Cohen, “Poésie et motivation ", dans Poétique, Paris, 1972, n 4 , p. 432.

4. Roman Jakobson, "Linguistique et poétique" dans Essais de linguistique générale, traduit par Nicolas Ruwet, Paris. 1963. p. 220. Nous soulignons.

5. Henri Mitterand, "Structuralisme linguistique et critique littéraire", dans SainteBeuve et la critique littéraire contemporaine - Acte du colloque de Liege, Les Belles-Lettres, 1972, p. 175.

6. Daniel Delas et Jacques Filliolet, $p$ p. cit., p. 111.

7. Alain Grandbois, Poèmes, les lles de la nuit, Rivages de l'homme, l'Étoile pourpre, Montréal, Hexagone, 1963, p. 55-56.

8. S. R. Levin, aLinguistic Structures in Poetry, La Haye, Mouton, 1964, cité par J. M. Adam, "Sur cinq vers, de Mystere de la parole: lire aujourd'hui "Neige" d'Anne Hébert", dans Études littéraires, Québec, Les Presses de I'Université Laval, décembre 1972, vol. V, n³, p. 464. 
9. Alain Grandbois, Poèmes, p. 62.

10. Lucien Tesnière, Éléments de syntaxe structurale, Paris, Klincksieck, 1969 cité par Lelia Picabia, "le Structuralisme", dans le Langage, Paris, Centre d'étude et de promotion de la lecture, "Les dictionnaires du savoir moderne», 1973, p. 499.

11. N.B. Les connecteurs utilisés dans cette étude sont les suivants: A-B indique une équivalence; $A\lrcorner B$ indique une non-équivalence; $A$ vs $B$ indique une non-équivalence doublée d'une opposition.

12. Daniel Delas et Jacques Filliolet, op. cit., p. 92.

13. Lucien Tesnière, op. cit.

14. Daniel Delas et Jacques Filliolet, op. cit., p. 114

15. C'est-à-dire «injection de sèmes " (Jean Cohen, op. cit., p. 440).

16. R. L. Wagner et J. Pinchon, Grammaire du français classique et moderne, Paris, Hachette, 1962, p. $152, n^{\circ} 164$.

17. A. J. Greimas, Du sens, Paris, Seuil, 1970, p. 282-283, cité par J. Bonenfant, "Lecture structurale d'un poème de Fernand Ouellette", dans la Barre du jour, Montréal, printempsété $1973, n^{\circ} 39-40-41$, p. 23-24.

18. N.B. Les termes consacrés désignant les diverses allégeances critiques ne prennent jamais, à nos yeux, une acception péjorative. Face à la disparité des points de vue, nous gardons pour le moment l'objectivité de "celui qui renseigne " et refusons d'endosser cette univocité de polémiques qui est celle de M. Henri Mitterand quand il traite la stylistique de "faillite" et le positivisme historique et l'explication de texte «a la française" de "retardataires" ( "Structuralisme linguistique et critique littéraire", dans Sainte-Beuve et la critique littéraire contemporaine, p. 180). D'ailleurs, nous avons personnellement essayé d'illustrer la méthode "impressionniste" dans un numéro de CoIncidences ( Essai d'explication du poème «Paysage en deux couleurs sur fond de ciel" de Saint-Denys Garneau ", Ottawa, Éditions de I'Université d'Ottawa, janvier-février 1974, vol. IV, $n^{\circ} 1$, p. 54-62). Aujourd'hui, cette lecture structurale de Grandbois fait entendre un autre "son de cloche " qui n'a d'autres prétentions que son effort pédagogique et la contribution de son éclairage à la cause de l'archilecture du poème 18 , non circonscrite encore par aucune école critique.

19. Daniel Delas et Jacques Filliolet, op. cit., p. 124.

20. Ibid. p. 68 .

21. Alain Grandbois, Poèmes, p. 24 et 51.

22. Daniel Delas et Jacques Filliolet, op. cit., p. 181

23. Alain Grandbois, Poèmes, p. 194.

24. Ibid. p. 91.

25. Jean Cohen, op. cit., p. 445. 